\title{
Spray Drop Size Distribution and Velocity Distribution issued from a Prefilming Airblast Atomizer
}

\author{
Diego Ferrando*1, Lorenzo Palanti ${ }^{2}$, François-Xavier Demoulin ${ }^{1}$, Benjamin Duret $^{1}$, Julien \\ Reveillon $^{1}$ \\ ${ }^{1}$ CNRS CORIA UMR 6614, University of Rouen Normandie, France \\ ${ }^{2}$ Department of Industrial Engineering (DIEF), University of Florence, Italy \\ *Corresponding author email: diego.ferrando@coria.fr
}

\begin{abstract}
This work explore the possibility to determine the spray characteristics at early stage of the atomization process. This approach has been firstly proposed in [7] propose to characterize the liquid-gas surface in term of surface curvature distribution allowing the determination of which part of the surface is already representative of the final spray. This innovative approach is based on numerical simulation but could be extended in principle on experimental imagery techniques for instance. In this work, the methodology is extended to include surface velocity measurements to provide the joint distribution of the spray in term of diameter and velocity. The configuration is representative of an aeronautic injector used in the European project CHAiRLIFT and focus on the prefilming airblast atomization. Such an atomization process involves a wide range of length scales. The diameter of the aeronautic atomizer could be three orders of magnitude higher than the diameter of the smallest droplet. Therefore, performing a CFD simulation is neither a straightforward nor a cheap process computationally speaking. Thus, a workflow, which divides the atomizer in less complex processes, is presented in this work to make the simulation affordable in an industrial perspective. The objective is to compute the properties that determine the spray behavior for the following combustion process. The OpenFOAM library is used to perform the simulation. In addition to the numerical strategy to capture first steps of the atomization process the focus of our presentation will be on the post processing of the simulation to extract the main features of the spray. The results presented concern the drop size distribution of the spray and the joint velocity distributions of the droplets. The post processing methodology is based on an analysis of the surface density joint distribution for curvatures and velocity. We believe this methodology can be used to enrich the spray injection models for further reactive spray flame simulations.
\end{abstract}

\section{Keywords}

Airblast Atomization, Spray, Volume of Fluid, Drop Size Distribution, Curvature.

\section{Introduction}

Prefilming airblast nozzles are suited for atomizing liquid fuels in continuous-flow combustion systems, such as gas turbines, where high velocity air flows are available. In this kind of atomizers, the fuel is first spread out in a thin liquid sheet and then subject to the atomization provoked by the shear stress at the liquid gas surface due to the high-velocity air flows [1]. Prefilming airblast atomizers are widely used in the aeronautic field since they allow an excellent atomization of the liquid fuel.Unlike other kind of atomizers, prefilming airblast atomizers creates smaller droplets which evaporates faster than large droplets. Thus, they preserve well balanced fuel vapor - air mixing before reaching the flame front, allowing for a better controlled combustion[2].

The goal of this work is to perform a numerical simulation of the prefilming airblast atomizer able to characterize the spray. Then, these characteristics are used as spray injection data for large scale turbulent combustion simulation. This approach is an attempt to complete the 
experimental measurement of the spray that is usually done to get this kind of information : Spray size distribution and spray angles. Numerically, the ultimate goal is to compute the Drop Size Distribution (DSD) and the droplet velocity joint distribution. The challenge is to connect the experimental spray data generally obtained far away downstream of the injector with the numerical characterisation of the spray that is limited to the close vicinity of the injector due to the cost of such simulation. The main concept is to analyse the spray formation at an early stage of the atomization by characterizing the spray surface and to determine which part of this surface carry relevant spray information [7].

The present work is part of the CHAIRLIFT European project 831881 - CHAiRLIFT - H2020CS2-CFP08-2018-01 which study compact helical arranged combustors with lifted flames which is suited for small aeronautical gas turbines. The Figure 1 shown the CHAiRLIFT atomizer which is the same studied in [3].

The whole injection system is fed by a simplex atomizer which creates a spray with a hollow cone shape. Most of the droplets reach the wall of the prefilmer where a thin fuel film is developed. In this atomizer, the inner air flow has a swirl component meanwhile the outer air flow has no swirl. The fuel film is carried by the air flow and is atomized to produce small droplets.

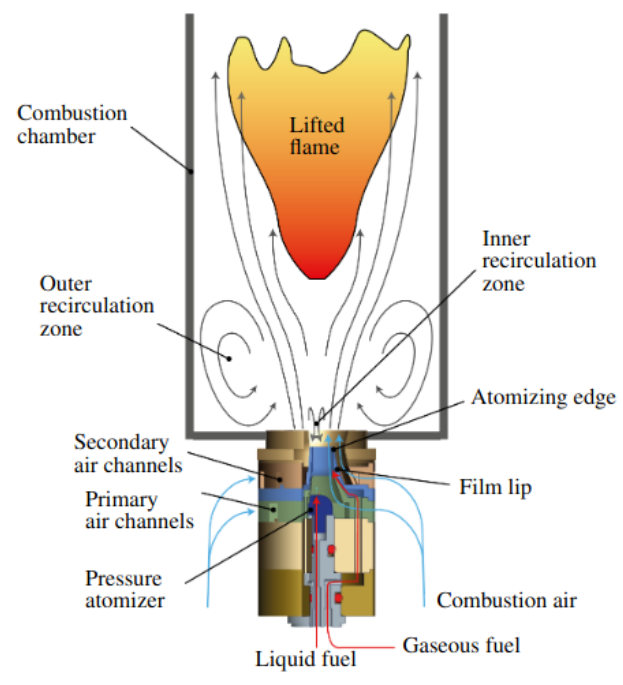

Figure 1. Schematic view of the CHAiRLIFT combustor [3]

Carrying out a single simulation to model the full airblast atomizer is still very time consuming and expensive computationally speaking. Due to the wide range of scale involved it cannot be achieved yet despite the CPU resources to our disposal . Indeed, the smallest droplet diameter could be three or four orders of magnitude smaller than the airblast nozzle diameter. Since the size of the computational cell have to be smaller than the smallest droplet to capture accurately the surface interface between the liquid and the air [11], performing a full domain simulation requires a large computational mesh [10]. Therefore, a modelling workflow has been defined to handle every part of the prefilming airblast atomizer separately. Then the last simulation concerning the liquid film atomization is analysed in details to provide spray characteristics. Nowadays, numerical post-processing techniques to determine the droplet characteristics are based on interface capturing techniques then the droplets are identified and characterized one by one [4][5][6]. These approaches are able to compute the droplet and velocity distribution accurately, but they demand an important computational resources; they require to simulate the spray formation until the end of the atomization process. On the other hand, the postprocessing technique proposed in [7] use only surface based information to compute the drop size distribution at early stage of the atomization process. In the present work, this approach is developed a step further to compute simultaneously the joint distribution of diameter and velocity of the spray. 


\section{Prefilming airblast modelling workflow}

The workflow proposed is shown in the Figure 2. The point 1 is the modelling of the pressure swirl atomizer. The spray angle is obtained from this simulation. The liquid film thickness is unknown, therefore it has to be estimated (point 2). Hence, a 2D simulation is carried out for this purpose and the length of this computational domain is calculated with the spray angle computed in the previous step.

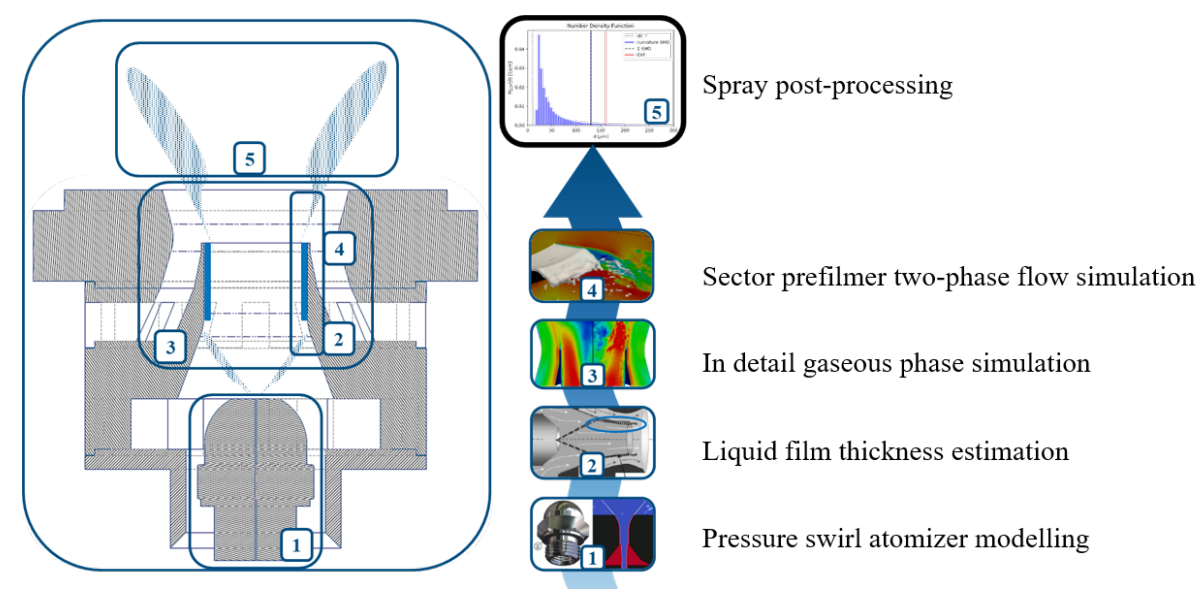

Figure 2. Prefilmer airblast atomizer modelling workflow

Following the work done by [7][9] in the planar prefilmer test case [12] a reduce angular sector numerical domain is simulated. To characterise the inlet velocity boundary condition for the gaseous phase, a single phase simulation is carried out to sample the velocity field (point 3 ) at different time steps to, afterwards, map it as transient Dirichlet boundary condition for the prefilmer simulation. Then the prefilmer has been simulated on a restricted angular sector of 8 degrees. At run time, the necessary variables for post processing analysis are stored on transverse measurement planes (point 5).

\section{Numerical methodology}

The OpenFOAM library is used to perform the simulation, specifically, the well konwn inter Foam solver. It offers an interface capturing method mostly based on the simulation of the liquid volume fraction by preserving a sharp transition between liquid and gas phase with a special sharpening numerical method used to limit the numerical diffusion. Time varying velocity is set up for the gaseous inlet velocity from an external simulation to reproduce accurate turbulent boundary condition. Meanwhile, a constant value velocity is set up for the fuel preserving the actual mass flow rate. A no-slip boundary condition has been used for the prefilmer walls and atmospheric pressure has been set up for the external boundaries. Finally, the two patches limiting the domains are related through periodic boundary conditions. The computational mesh is shown in the Figure 3. It has $8 \mathrm{M}$ cells and the zone where data are sampled for the post processing is particularly refined. The Smagorinsky turbulence LES sub-model has been used in this study. Concerning the numerical setup, a PIMPLE algorithm is employed and coupled with the liquid volume fraction equation. Second-order schemes are used both in space and time. Variable time-stepping is used, the maximum CFL number is limited to 0.4 . The computation has ran for 10 days using $240 \mathrm{CPU}$ having a total of $60 \mathrm{~K}$ of computational hours.

\section{Sampling and Post-processing methodology}

This technique is based on the measurement of the interface between the liquid and the gas to extract its characteristics in term of velocity and curvature. These information are then analyse to characterize the droplets. In other words, every variable sampled is weighted by the amount of surface (area). The sampled variables are the surface interface density flux $(\phi \Sigma=\mathbf{u} \cdot \mathbf{n}|\nabla \alpha|)$, 

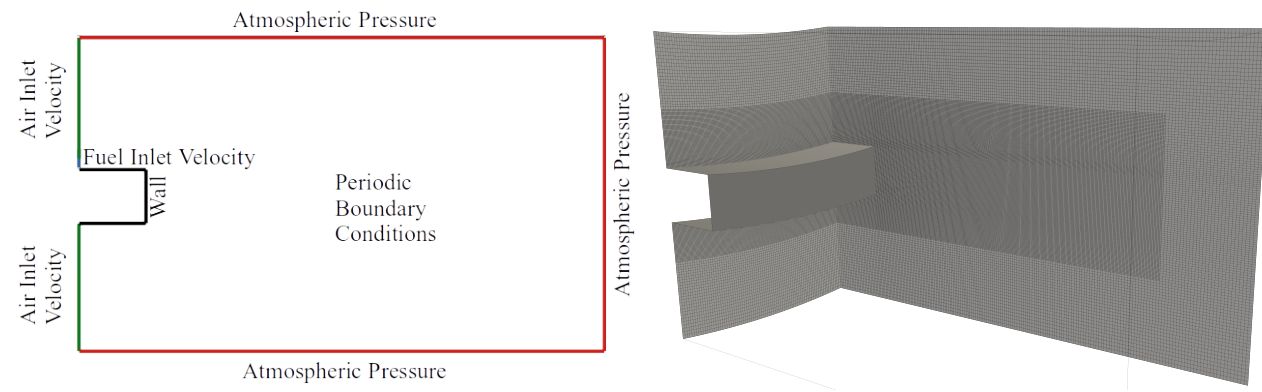

Figure 3. Simulation set-up and computational mesh

the curvature $(\kappa=\nabla \cdot(\nabla \alpha /(|\nabla \alpha|)))$, the velocity vector $(\mathbf{u})$ and liquid volume fraction flux $(\phi \alpha=\mathbf{u} \cdot \mathbf{n} \alpha)$. These variables are stored at determined coordinates and at every time step. To sample the data a measurement zone is set characterized by it surface with its normal directed to stream-wise direction. All fluxes going through the surface have to be recorded but keeping the local information. Thus, this surface is filled by a set of probes, having one probe on every face of the numerical cell composing the surface.

\section{Sauter Mean Diameter results}

The Sauter mean diameter (SMD) represents the ratio of the volume to the surface of a spray but this notion can be extended even for a general liquid-gas flows composed of liquid structured of any shape: (Equation 1).

$$
D_{32}=\frac{\sum_{i} D_{p, i}^{3}}{\sum_{i} D_{p, i}^{2}}=6 \frac{V_{l}}{A_{l}}[1]
$$

Thus, the SMD can be computed for a liquid-gas flow crossing a surface and averaged in time following the Equation 2 that is computed using the stored variables over the measurement surface.

$$
D_{32}(S)=6 \frac{\int_{T} \iint_{S}(\alpha \boldsymbol{u} \cdot \boldsymbol{n} d s) d t}{\int_{T} \iint_{S}(\Sigma \boldsymbol{u} \cdot \boldsymbol{n} d s) d t}[7]
$$

The Figure 4 shows the evolution of the SMD along the axial axis starting from the edge of the prefilmer. The value calculated at the lip of the atomizer is not a representation of any characteristic diameter but a characteristic length. The SMD first increases up to a maximum value where some liquid is accumulated increasing the thickness of the liquid sheet just before being atomized. Then, the SMD strongly decreases due to an active primary break-up that generates smaller liquid elements. Finally, this value remains constant, which is an indication that most of the primary break-up has been taken place even if there are some ligaments or other liquid parcel with complex surface morphology that are not yet fully atomized.

\section{Surface Curvature Distribution (SCD)}

From a known cloud of droplets (drop size distribution) is it possible to obtain the surface curvature distribution[8] since the two distribution are linked by the relationship between the diameter and the curvature. In the reverse way, it is possible to compute the drop size distribution from the surface curvature distribution. From the sampled data, the total amount of surface interface is stored in different types of curvature obtaining the surface curvature distribution.

Not all the curvature samplings are representatives of the final droplet, thus, the distribution has to be cleaned up to distinguish which surface elements has to be preserved. In the Figure 5 it can be observed that there are different kinds of structures. Starting from the left side of distribution, it can be seen that there is surface area which is related to a negative curvature. 


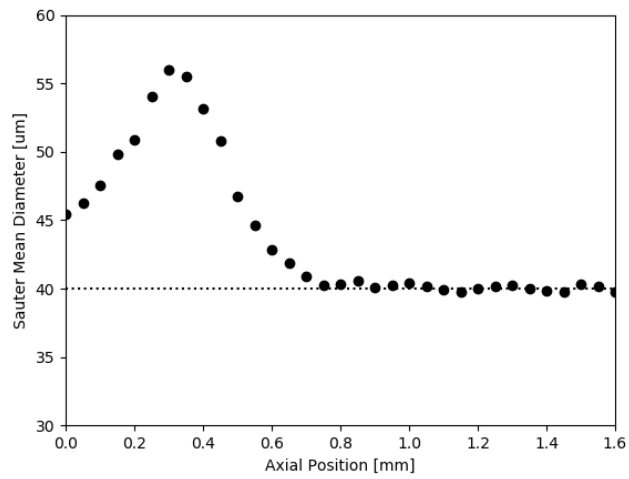

Figure 4. Sauter Mean Diameter computed along the axial axis

This liquid structures correspond to concave elements such a bubbles, ligaments or surface waves (capillary waves for instance). As a preliminary post-process, all the surface related to a negative value is removed since that structures are definitely not droplets. The not considered surfaces (negative curvatures) are shown in black and it correspond to $11.3 \%$ of the total surface sampled.

\section{Drop Size Distribution (DSD)}

Once the surface curvature distribution is cleaned up, the drop size distribution is computed by the relation between the curvature and the diameter $\left(d_{\kappa}=4 / \kappa\right)$ and the relation between the surface interface and the number of droplets $\left(n\left(d_{\kappa}\right)=A_{\kappa} / \pi d_{\kappa}\right)$. These relations are based on the most simple assumption that the retained surface correspond to shell piece of the final droplets. Many other possibilities could be considered but for the time being we have kept this simple approach corresponding also to the approach developed in [7].

The Figure 6 shows the drop size distribution computed from the surface curvature distribution. We can observe that there are a small number of large droplets. They are non-physical droplets since they correspond to a bigger liquid structures such as ligaments or almost flat structures. To discard this very large diameters, the distribution is clipped to match the SMD estimated through the global relation Equation 2. This procedure proposed in [7] enforced the linked between local surface curvature measurement and the global characteristic of the spray that are also computed within the numerical simulation keep a self-consistent approach.

The result of this methodology can be observed on Figure 5. The last figure shows the SCD where the negative curvatures and the party corresponding to non-physical large droplets have been discarded. On the right hand side, the iso-surface of the liquid volume fraction is drawn colored by the curvature where the surface have been retained. All the discarded structures corresponding to the surface element still under atomization process before, i.e. that have not yet reach there final state where they can be considered has representative of the final spray, are represented in black. As expected close to the injector lip most of the surface is blacked since the atomization has not started yet, but farther downstream more and more colour surface element appear as the primary break up and the full atomization process takes places. At the transverse plane location, where the global SMD is stable and the curvature distribution used to estimate the SMD is measured, the surface kept to compute the DSD is $54 \%$ of the total surface.

\section{Velocity joint distributions}

In a similar way, the velocity joint distributions can be computed. The number of droplets is associate with their velocity vector. In the Figure 7 the three velocity component distributions are shown. The dotted line is the inlet fuel velocity meanwhile the straight line is the inlet air 

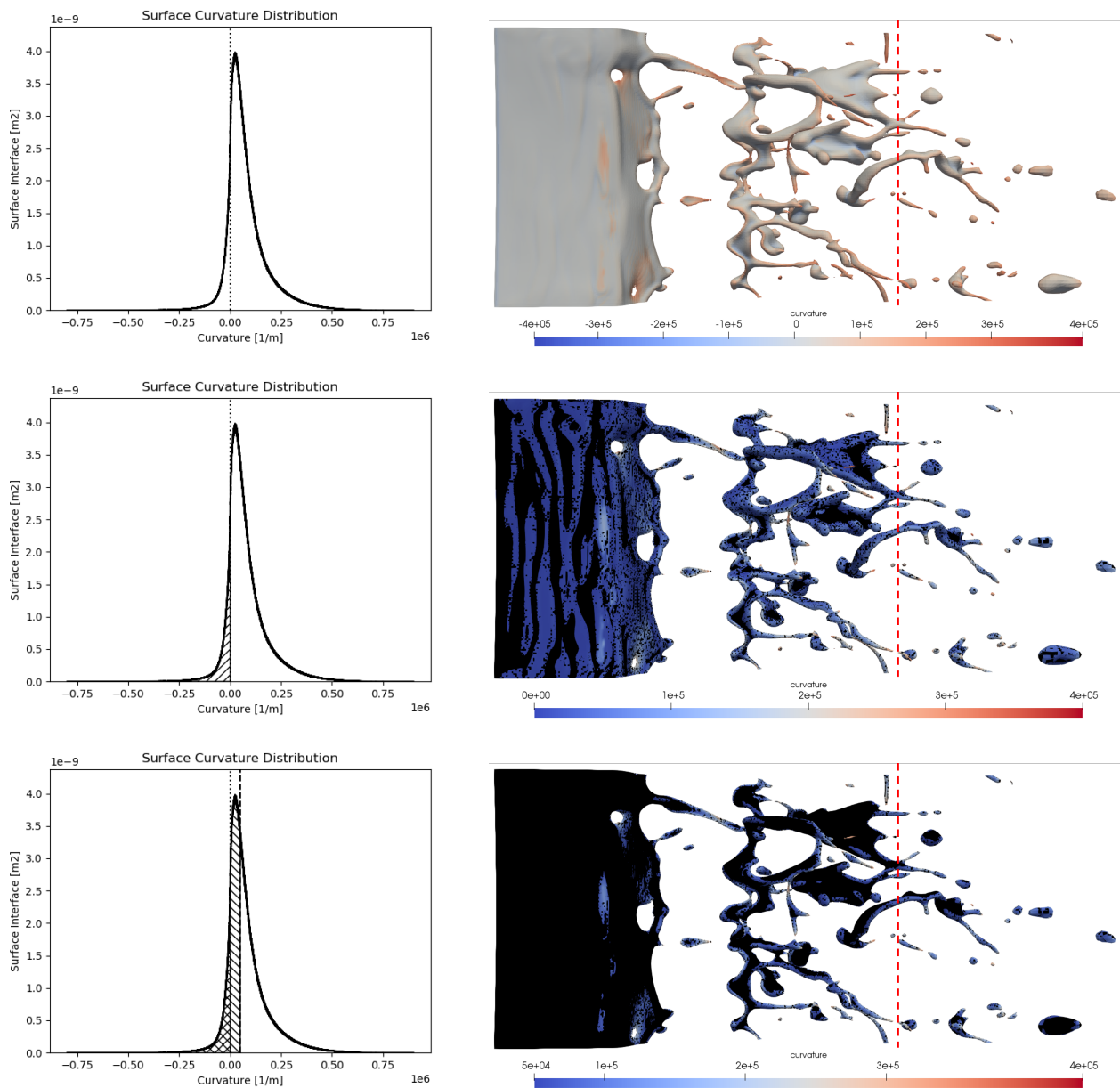

Figure 5. Surface Curvature Distribution (Left) and $\alpha$ contours (right) displaying the removed surface each step of the post-processing. From top to bottom: None surface removed, negative curvature surfaces removed and small curvature surfaces removed. Red dotted line represents the post-processing measurement plane.

velocity averaged on time and space. As expected, the estimated droplets are accelerated from their initial velocity but not overcoming the air velocity. This fact does not happen in the radial component distribution but still the result is physical since once the fuel have left the prefilmer wall, the tangential component of the liquid structures is converted into the radial component. It is also possible to compute the drop velocity distribution for each kind of droplet diameter. It can be noticed in Figure 8 that the smaller droplets $(D=[10-11] \mu m)$ are more accelerated by the air flow than the medium droplets $(D=[40-41] \mu m)$ and the bigger droplets $(D=$ $[70-71] \mu m)$. This effect is expected due to the lower inertia of the smaller droplets compared to the bigger ones and also to the momentum conservation.

\section{Conclusions}

A workflow to carry out the modelling of an industrial prefilming airblast atomizer has been proposed. The computational resources has been greatly reduced in contrast to perform one single simulation that encompasses all the physical phenomena taking place and dividing them in different length scales.

The innovative post-processing technique proposed by [7] has been further developed using a CFD simulation based on OpenFoam that include an interface capturing method. It has been reported that it is possible to extract from the prefilmer sector simulation the surface curvature distribution. Then, a self-consistent analysis allows to estimate the final drop size distribution at early stage of the atoimization process. The new contribution to this post-processing methodology concerns the calculation of the joint distribution for the three velocity components and 

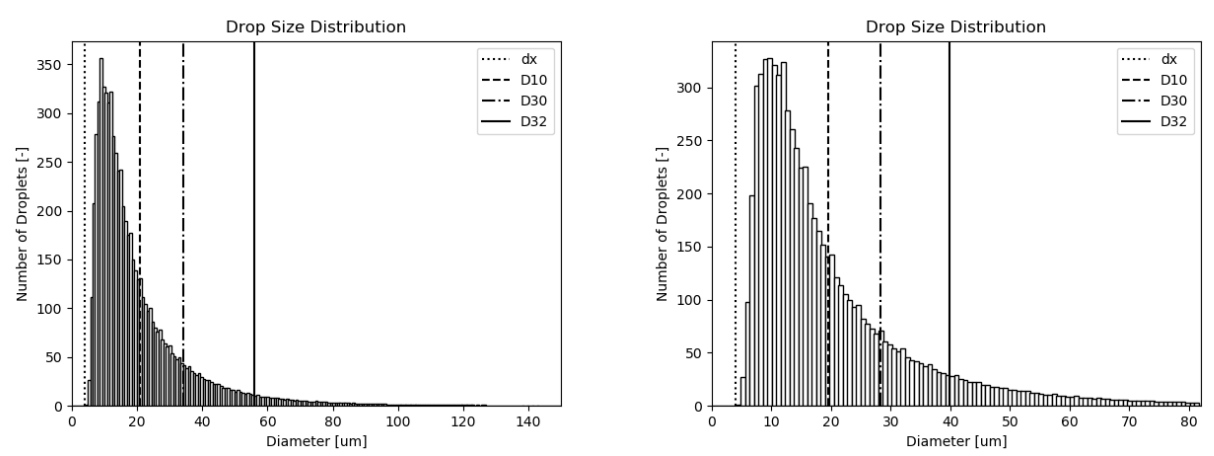

Figure 6. Drop Size Distribution: before clean up (left) and cleaned up(right)
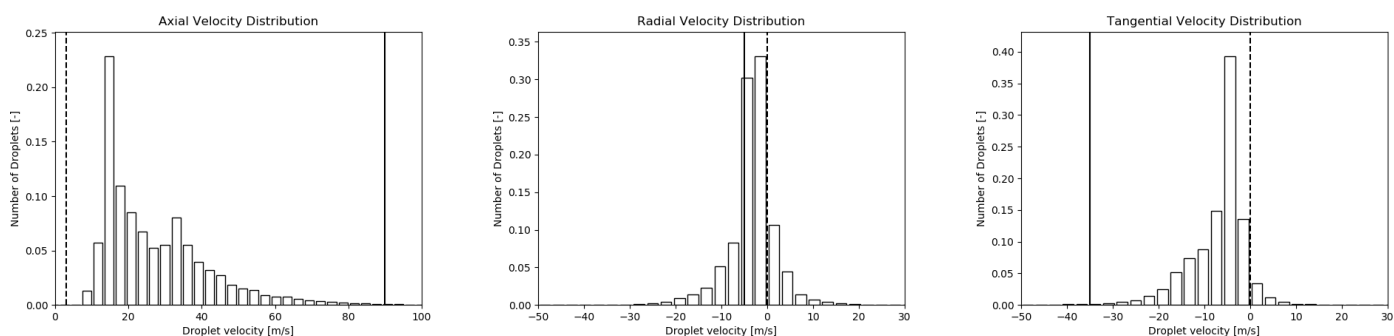

Figure 7. Velocity joint Distribution. Left to right: axial, radial and tangential component
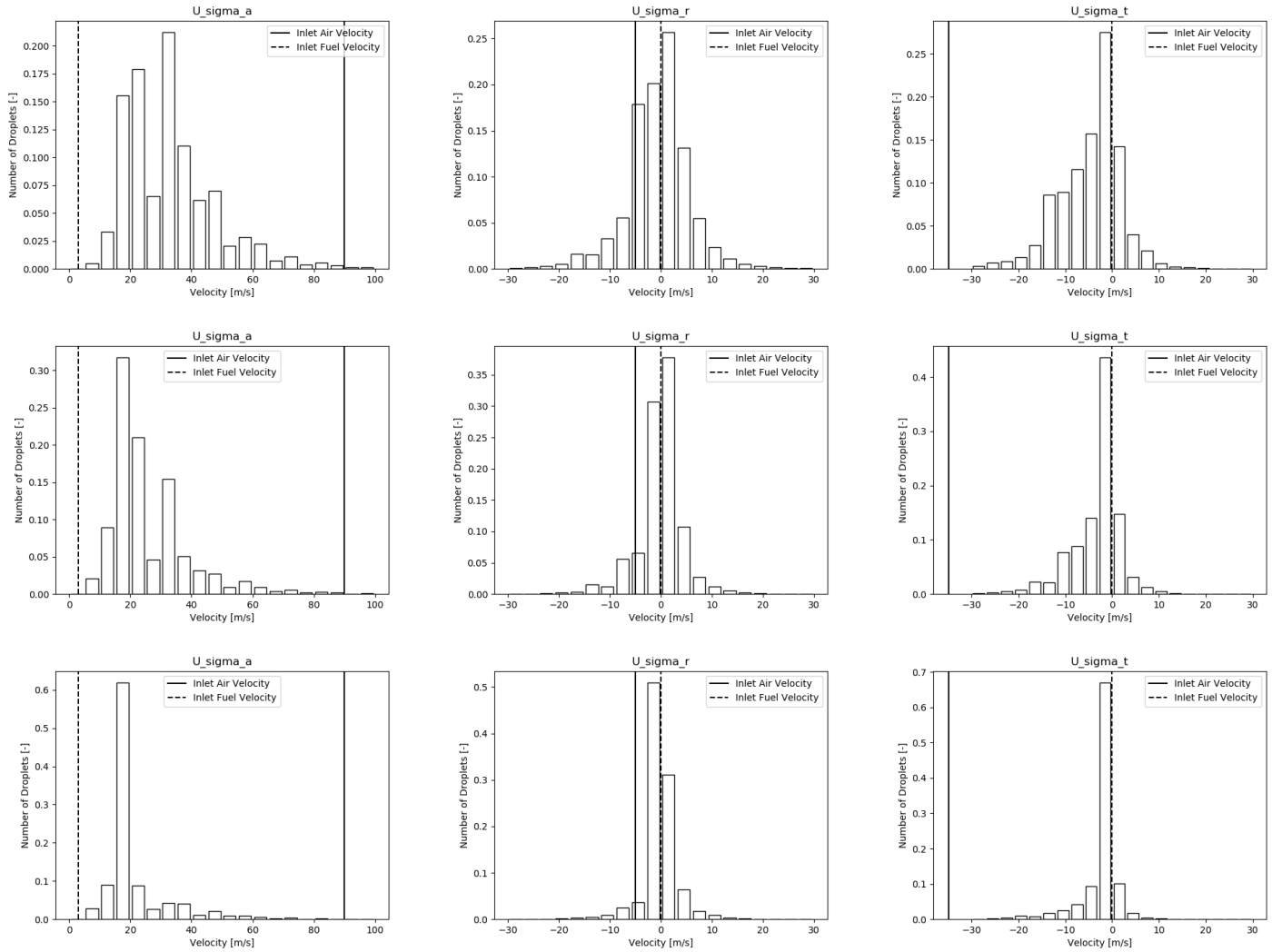

Figure 8. Velocity joint Distribution by droplet diameter. Left to right, axial, radial and tangential component. Top to bottom: small, medium and big droplets 
the droplet diameter. Hence, the spray injection characteristics can be determined giving the opportunity to use these data to set-up the injection model for further reacting flow simulation which is the purpose of this work inside the CHAIRLIFT project.

\section{Acknowledgements}

This work was granted access to the HPC resources of IDRID, TGCC and CINES under the allocation A0072B06153 and A0092B06153 made by GENCI (Grand Equipement National de Calcul Intensif) and also for the computing time at CRIANN (Centre Régional Informatique et d'Applications Numériques de Normandie) under the scientific project No. 2006011. This work has been also founded by the CHAIRLIFT European project 831881 - CHAiRLIFT - H2020CS2-CFP08-2018-01.

\section{Nomenclature}

$\alpha \quad$ Liquid Volume Fraction [-]

$\Sigma \quad$ Surface Interface Density $\left[\mathrm{m}^{-1}\right]$

$\phi \quad$ Flux $\left[m^{3} s^{-1}\right]$

$\kappa \quad$ Curvature $[m-1]$

$D_{32} \quad$ Sauter Mean Diameter $[m]$

$\boldsymbol{u} \quad$ Velocity vector $\left[\mathrm{ms}^{-1}\right]$

$d_{k} \quad$ Diameter related to a curvature $[\mathrm{m}]$

$n\left(d_{k}\right) \quad$ Number of droplets related to a curvature [-]

$A_{k} \quad$ Surface area related to a curvature $\left[\mathrm{m}^{2}\right]$

\section{References}

[1] Lefebvre, A. H., McDonell, V. G., 2017, "Atomization and Sprays". CRC Press.

[2] Chaussonnet, G., Riber, E., Vermorel, O., Cuenot, B., Gepperth, S., Koch, R., 2013, ICLASS 2013, 13th Triennial International Conference on Liquid Atomization and Spray Systems.

[3] Sedlmaier, J., Habisreuther, P., Zarzalis, N., Jansohn, P., 2014, Proceedings of ASME Turbo Expo 2014: Turbine Technical Conference and Exposition.

[4] Braun, S., Wieth, L., Holz, S., Dauch, T.F., Keller, M.C., Chaussonnet, G., Gepperth, S., Koch, R., Bauer, H-J., 2019, International Journal of Multiphase Flows, 114 , pp. 303-315.

[5] Warncke, K., Gepperth, S., Sauer, B., Sadiki, A., Janicka, J., Koch, R., Bauher, H.-J., 2017, International Journal of Multiphase Flows, 91 , pp. 208-224.

[6] Mukundan, A.A., Ménard, T., Berlemont, A., Brändle de Motta, J. C., 2019, ICLASS 2019, 29th Triennial International Conference on Liquid Atomization and Spray Systems.

[7] Palanti, L., Puggelli, S., Langone, L., Andreini, A., Reveillon, J., Duret, B., Demoulin, F.X., 2021, International Journal of Multiphase Flows, xx (x), pp. xxxxxxx.

[8] Canu, R., Puggelli, S., Essadki, M., Duret, B., Menard, T., Massot, M., Reveillon, J., Demoulin, F.X., 2018, International Journal of Multiphase Flows, 107 , pp. 230-245.

[9] Sauer, B., Sadiki, A., Janicka, J., 2014, The Journal of Computational Multiphase Flows, 6(3), pp.179-192.

[10] Li, X,. Soteriou, M. C., Kim, W., Cohen, J. M., 2014, Journal of Engineering for Gas Turbines and Power, 136, 36(7): 071503 (10 pages).

[11] Anez, J., Ahmed, A., Hecht, N., Duret, B., Reveillon, J., Demoulin, F.X., 2019 International Journal of Multiphase Flows, 113, pp. 325-342.

[12] Gepperth, S., Guildenbecher, D., Koch, R., Bauer, H.-J., 2010, ICLASS 2010, 10th Triennial International Conference on Liquid Atomization and Spray Systems. 